\title{
THE PATHOLOGICAL EFFECT OF THE BORDETELLA DERMONECROTIC TOXIN IN MICE
}

\author{
T. MAGYAR ${ }^{1 *}$, R. GlÁVITS ${ }^{2}$, G. D. PUllinger ${ }^{3}$ and A. J. LAX ${ }^{3}$ \\ ${ }^{1}$ Veterinary Medical Research Institute, Hungarian Academy of Sciences, H-1581 \\ Budapest, P.O. Box 18, Hungary; ${ }^{2}$ Central Veterinary Institute, Budapest, Hungary; \\ ${ }^{3}$ Oral Microbiology, Guy's, King's and St Thomas' Dental Institute, \\ King's College London, London, United Kingdom
}

(Received October 9, 2000; accepted October 17, 2000)

\begin{abstract}
The effect of dermonecrotic toxin (DNT) expression of Bordetella bronchiseptica was studied in mice by comparing the pathology induced by a wild type strain with that induced by an isogenic DNT- strain in which part of the structural gene has been replaced by an antibiotic resistance cassette. While extracts of strain B58 proved toxic in intravenously inoculated mice, similar extracts from strain B58GP had lost toxic activity. The parent (B58) and the mutant (B58GP) strains of B. bronchiseptica each possessed comparable virulence for mice. These findings confirmed that DNT production was successfully abolished in strain B58GP while other virulence characteristics required for pathogenicity in mice remained intact, at a comparable level to the parent strain. Turbinate atrophy was observed in mice infected with the DNT+ strain, but not in those infected with the DNT- strain. This indicates that DNT is the cause of turbinate atrophy in the mice and not other factors produced by phase I strains of B. bronchiseptica. $B$. bronchiseptica DNT showed a lienotoxic effect (lymphocyte depletion and a reduction in the intensity of extramedullar haemocytopoieis) that is considered to adversely alter the immune function of the host animal. In mice infected with strain B58GP, catarrhal pneumonia with characteristic lympho-histiocytic peribronchial and perivascular infiltration was noticed. In mice infected with strain B58, large necrotic areas were seen surrounded by an inflammatory reaction. The DNT appears to directly damage lung tissues, at least in mice. DNT production seems to enhance the establishment of $B$. bronchiseptica in the lungs, presumably by reducing the local resistance and causing severe local damage to the lung tissues.
\end{abstract}

Key words: Bordetella bronchiseptica, dermonecrotic toxin, mouse

Bordetella bronchiseptica is a widely prevalent respiratory pathogen associated with atrophic rhinitis (AR) of swine, tracheobronchitis (kennel cough) in dogs, and respiratory infections in other domesticated animals such as rabbits (Goodnow, 1980). Its phase I strain produces a range of potential virulence de-

*E-mail: tibor@novell.vmri.hu; Fax: +36 (1) 467-4076 
terminants, including the adhesins: filamentous haemagglutinin, pertactin, and the toxins: adenylate cyclase-haemolysin toxin, tracheal cytotoxin and dermonecrotic toxin (DNT). Although the role of B. bronchiseptica virulence determinants has not been elucidated satisfactorily, it seems that colonisation of the nasal cavity in large numbers and the DNT-producing ability of the strains are the most important factors in the pathogenesis of turbinate atrophy caused by B. bronchiseptica in swine. The crucial role of B. bronchiseptica DNT in producing turbinate hypoplasia has long been suspected (Roop et al., 1987; Magyar et al., 1988), however, these studies used naturally isolated DNT-negative strains and so the possibility remained that other factor(s) not detected in vitro could have been responsible for turbinate atrophy. The difficulty in producing sufficient quantities of highly purified DNT or a DNT-negative isogenic mutant strain has hindered the elucidation of the exact role of DNT in the pathogenesis of turbinate atrophy. The construction of an isogenic mutant strain, in which the expression of DNT was eliminated paved the way for assessing the contribution of DNT to the virulence of $B$. bronchiseptica in swine.

Although the mouse is not a natural host of B. bronchiseptica it can be used to characterise virulence in several ways. Krüger and Horsch (1982) described a mouse lienotoxicity test with broth cultures for determining the toxinproducing ability of $B$. bronchiseptica in intravenously inoculated mice. Strains that were lethal to mice and which caused atrophy of the spleen at sublethal doses were considered toxic. In contrast, atoxic strains did not cause death and induced significant hypertrophy of the spleen. Later, it was demonstrated that only cell-free sonicated extracts (SE) were suitable for accurate determination of the DNT-producing ability of $B$. bronchiseptica while whole-cell suspensions (WCS) could be used to assess the virulence of the strain independent of its DNT production (Magyar, 1990). Intranasal inoculation of new-born mice proved to be suitable for modelling the development of turbinate atrophy (Sawata and Kume, 1982; Magyar et al., 1985; Kielstein et al., 1987). Lesions similar to those described in AR affected pigs were found in the nasal cavity of mice inoculated with DNT-producing phase I strains of $B$. bronchiseptica at the age of two days and examined histopathologically three weeks later.

The present report gives a preliminary characterisation of an isogenic pair of DNT+ and DNT- strains of B. bronchiseptica in mice, and demonstrates that DNT is responsible for much of the pathology induced by $B$. bronchiseptica infection of mice. 


\section{Materials and methods}

\section{Bacterial strain}

B. bronchiseptica strain B58 was isolated from a herd with clinical AR. It is a virulent strain that is toxigenic in mice and was shown to express phase I virulence factors (Magyar et al., 1988).

\section{Construction of the B58GP strain}

The construction of the B58GP mutant will be described elsewhere. Briefly, an internal fragment of the cloned DNT gene was deleted, and a kanamycin resistance cassette inserted in its place. This was transferred into B. bronchiseptica $\mathrm{B} 58$ by allelic exchange.

\section{Haemagglutination test}

A slide agglutination test was used (Semjén and Magyar, 1985). B. bronchiseptica strains were cultured on BG agar for $24 \mathrm{~h}$ at $37^{\circ} \mathrm{C}$ and their haemagglutination (HA) activity was examined with $5 \%$ calf erythrocytes.

\section{Testing virulence and toxicity}

Virulence and toxicity were tested in the mouse spleen toxicity (lienotoxicity) test as described elsewhere (Magyar, 1990). Briefly: whole-cell suspensions and cell-free sonicated extracts were prepared for testing virulence and toxicity, respectively. Groups of five white CFLP (LATI, Gödöllö, Hungary) mice weighing 16-18 g were inoculated intravenously with $0.2 \mathrm{ml}$ of one of the serial threefold dilutions of the test materials. Deaths were recorded up to 7 days post inoculation when the survivors were killed and spleen masses were determined. For comparison, relative spleen masses were calculated for $10 \mathrm{~g}$ body mass [spleen mass $(\mathrm{mg})$ per body mass $(\mathrm{g}) \times 10$ ].

\section{Infection of mice}

Two groups of five and one group of three litters were used in the study. Group 1, which consisted of 51 new-born mice, was inoculated with B. bronchiseptica B58. Group 2, which consisted of 50 new-born mice, was inoculated with B. bronchiseptica B58GP. The 27 mice of Group 3 served as untreated control.

B. bronchiseptica strains cultured on BG agar at $37^{\circ} \mathrm{C}$ for $24 \mathrm{~h}$ were diluted in phosphate-buffered saline (PBS), $\mathrm{pH} 7.2$ to give an approximate concentration of $5 \times 10^{6}$ colony forming units $(\mathrm{cfu}) / \mathrm{ml}$. Mice were inoculated at 1-2 days of age by dropping $0.003 \mathrm{ml}$ of bacterial suspension into each nostril. Mice were killed by ether overdose at 21 days post infection and subjected to postmortem examination. The right lung lobes of two randomly selected mice from each litter were taken for 
bacterial culture; samples of nose, lung and spleen tissues were fixed in $10 \%$ neutral buffered formalin for histopathological examination.

\section{Statistical analysis}

Lethality in intranasally inoculated mice was analysed with the TukeyKramer Multiple Comparisons Test.

\section{Results}

Properties of the strains of $\mathrm{B}$. bronchiseptica

Both strains (B58 and B58GP) produced haemolytic colonies on BordetGengou agar and agglutinated calf erythrocytes, confirming the phase I status of both strains.

Results of the mouse lienotoxicity test are shown in Table 1. The WCS and cell-free SE of B. bronchiseptica B58 were lethal to mice and induced a reduction in spleen mass when given at sublethal doses. B. bronchiseptica B58GP inoculated in living form was as lethal as the WCS of the parent strain but the surviving mice showed considerable splenic hypertrophy. SE of B58GP were not lethal and produced a slight increase in spleen mass.

\section{Table 1}

Mouse lienotoxicity test with whole-cell suspensions (WCS) and cell-free sonicated extracts (SE) of Bordetella bronchiseptica strains B58 and B58GP

\begin{tabular}{|c|c|c|c|c|c|c|c|c|}
\hline \multirow{3}{*}{ Dilution } & \multicolumn{4}{|c|}{ B58 } & \multicolumn{4}{|c|}{ B58GP } \\
\hline & \multicolumn{2}{|c|}{ WCS } & \multicolumn{2}{|c|}{ SE } & \multicolumn{2}{|c|}{ WCS } & \multicolumn{2}{|c|}{ SE } \\
\hline & let & $\mathrm{rsm}$ & let & rsm & let & $\mathrm{rsm}$ & let & $\mathrm{rsm}$ \\
\hline UD & $5 / 5$ & - & $5 / 5$ & - & $5 / 5$ & - & $0 / 3$ & 64 \\
\hline 3 & $5 / 5$ & - & $5 / 5$ & - & $5 / 5$ & - & $0 / 3$ & 69 \\
\hline 9 & $3 / 5$ & $38^{\mathrm{a}}$ & $0 / 5$ & 32 & $5 / 5$ & - & $0 / 3$ & 58 \\
\hline 27 & $0 / 5$ & 44 & $0 / 5$ & 28 & $0 / 5$ & 136 & ND & ND \\
\hline 81 & $0 / 5$ & 53 & $0 / 5$ & 49 & $0 / 5$ & 103 & ND & ND \\
\hline
\end{tabular}

let $=$ lethality $($ died/total); $\mathrm{rsm}=$ relative spleen mass [calculated for $10 \mathrm{~g}$ body mass: spleen mass $(\mathrm{mg})$ per body mass $(\mathrm{g}) \times 10]$; $\mathrm{UD}=$ undiluted; $\mathrm{ND}=$ not determined; ${ }^{\mathrm{a}}$ relative spleen mass of the control $=59$ (five control mice were inoculated with PBS wash of an uninoculated BG plate)

\section{Infection of mice}

Table 2 shows the results of the $B$. bronchiseptica infection in the newborn mice during the three weeks of the observation period. The untreated control mice all survived and B. bronchiseptica was not isolated from these animals. In contrast, almost half of the mice died in both infected groups. Lethality was 
therefore independent of the DNT production of the B. bronchiseptica strains used for infection. On the other hand, about 20-fold less B. bronchiseptica $\mathrm{B} 58 \mathrm{GP}$ was recovered from the lungs than $B$. bronchiseptica $\mathrm{B} 58$.

\section{Table 2}

Average number of deaths during the observation period and the average number of Bordetella bronchiseptica bacteria isolated from the lungs

\begin{tabular}{lccc}
\hline Group & Average litter size & Average $\%$ of mice died & Average cfu of $\mathrm{Bb}^{*}$ \\
\hline 1 (B58) & 10.2 & $48.1^{\mathrm{a}}$ & $3.7 \times 10^{4}$ \\
2 (B58TM) & 10.0 & $44.0^{\mathrm{a}}$ & $7.8 \times 10^{5}$ \\
3 (Control) & 9.0 & $0^{\mathrm{b}}$ & 0 \\
\hline
\end{tabular}

*Average number of Bordetella bronchiseptica per g lung tissue; ${ }^{\mathrm{a}, \mathrm{b}}$ Values within the column with different superscripts are significantly different $(\mathrm{p}<0.05)$; $\mathrm{cfu}=$ colony forming unit; $\mathrm{Bb}=$ Bordetella bronchiseptica

\section{Histopathological findings}

Table 3 summarises the incidence of characteristic lesions in the groups.

Infection with the DNT+ B58 strain produced the most severe lesions. Epithelial cells of the nasal turbinates were disarranged. Hyperplasia, dysplasia, and desquamation were detectable in the epithelial layer. The most obvious differences between this group and the others were the loss of cilia from epithelial cells and the resorption of bone resulting in turbinate atrophy that was observed only in mice infected with B58 (Figs 1, 2, 3 and 4). There was only slight lympho-histiocytic infiltration and accumulation of exudate without other lesions in the nasal turbinates of mice (Figs 3 and 4) infected with DNT-B58GP strain.

In the B58 infected group, large necrotic areas were observed in the lung (Fig. 5), and severe lymphocyte depletion was seen in the spleen (Fig. 6).

In the B58GP infected group, catarrhal pneumonia with characteristic lympho-histiocytic peribronchial and perivascular infiltration - without necrosis - occurred in the lungs (Fig. 7). Lymphoid hyperplasia and increase of intensity of extramedullar haemocytopoieis (Fig. 8) were detectable in the spleen.

\section{Discussion}

The Bordetella dermonecrotic toxin is a potent molecule that modifies and activates members of the Rho family of signalling proteins (Masuda et al., 2000). The Rho proteins are essential for regulation of the cell cytoskeleton, and chronic activation of these proteins has effects on many aspects of cell function, including intracellular signalling pathways and cell cycle progression (Ridley, 1996). Nevertheless it is unclear what role DNT plays in vivo, since mouse lethality has 
been shown to be unaffected by deletion of the DNT gene in B. pertussis (Weiss and Goodwin, 1989). The gene for B. bronchiseptica DNT has been cloned and expressed (Pullinger et al., 1996).

Table 3

Histopathological changes in mice inoculated with Bordetella bronchiseptica strains B58 and B58GP

\begin{tabular}{|c|c|c|c|}
\hline \multirow{2}{*}{ Lesions } & \multicolumn{2}{|c|}{ Strain } & \multirow{2}{*}{ Control } \\
\hline & B58 & B58GP & \\
\hline \multicolumn{4}{|l|}{ Nasal turbinates } \\
\hline - hyperplasia of epithelium & + & - & - \\
\hline - dysplasia of epithelium & + & - & - \\
\hline - loss of cilia from epithelial cells & + & - & - \\
\hline - resorption of bone & + & - & - \\
\hline - increased number of fibroblasts & + & - & - \\
\hline - turbinate atrophy & + & - & - \\
\hline - foci of lymphocytes and plasma cells & + & + & - \\
\hline - exudate in the scrolls of the turbinates & + & + & - \\
\hline \multicolumn{4}{|l|}{ Lungs } \\
\hline - necrotic pneumonia & + & - & - \\
\hline $\begin{array}{l}\text { - catarrhal pneumonia with lympho-histiocytic peribronchial } \\
\text { and perivascular infiltration }\end{array}$ & - & + & - \\
\hline \multicolumn{4}{|l|}{ Spleen } \\
\hline - lymphocyte depletion & + & - & - \\
\hline - decrease of intensity of extramedullar haemocytopoieis & + & - & - \\
\hline - lymphoid hyperplasia & - & + & - \\
\hline
\end{tabular}

We describe here the effect of DNT expression in a mouse model by comparing the pathology induced by a wild type B. bronchiseptica strain with that induced by an isogenic DNT - strain in which part of the structural gene has been replaced by an antibiotic resistance cassette. While extracts of strain B58 proved toxic in mice, similar extracts from strain B58GP had lost toxic activity. The parent (B58) and the mutant (B58GP) strains of B. bronchiseptica each possessed comparable virulence for mice. These findings confirmed that DNT production was successfully abolished in strain B58GP while other virulence characteristics required for pathogenicity in mice remained intact, at a comparable level to the parent strain. In a previous study (Magyar, 1990), a naturally found DNTnegative $B$. bronchiseptica strain designated PV6 behaved similarly in intravenously inoculated mice, and it was concluded that DNT did not affect the lethality of live cultures of $B$. bronchiseptica for mice. This hypothesis has been confirmed by the present results using an isogenic pair of DNT+ and DNT- strains of $B$. bronchiseptica. 

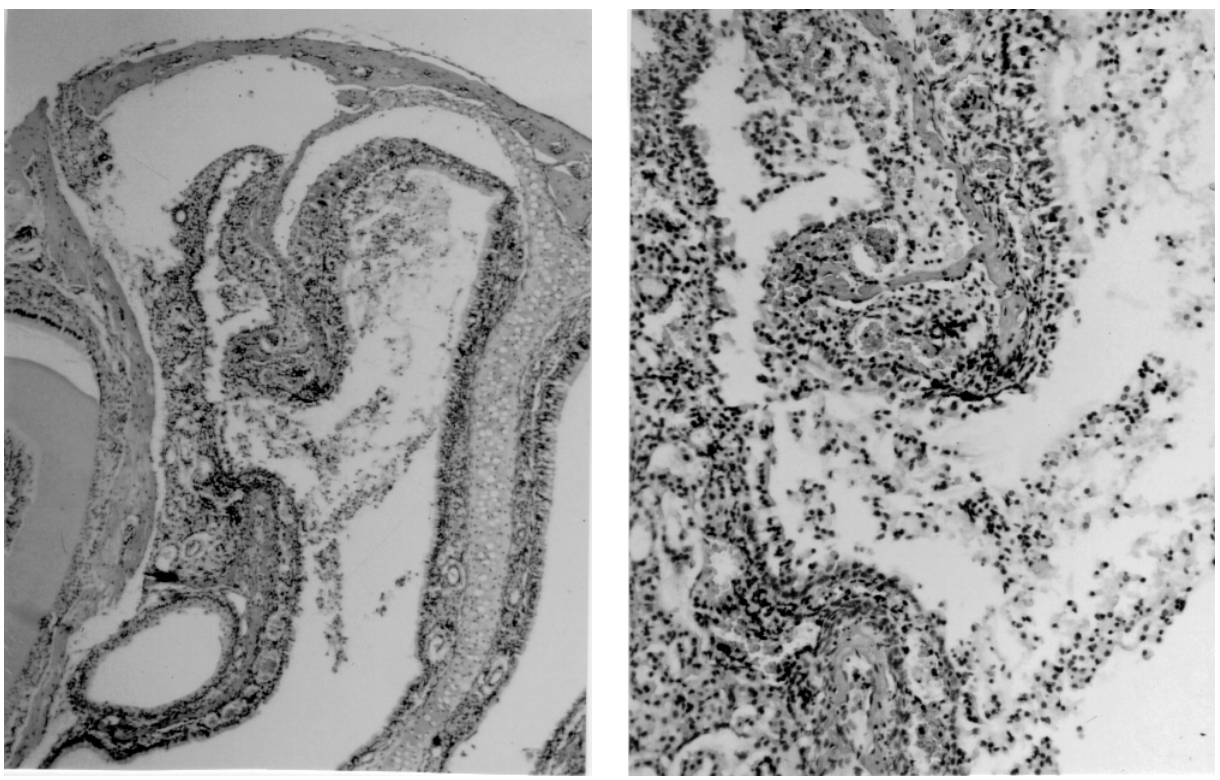

Figs 1 and 2. The nasal turbinates of a mouse infected with DNT+ B58 Bordetella bronchiseptica strain. Turbinate atrophy with epithelial lesions (loss of cilia, dysplasia, desquamation), resorption of bone and lympho-histiocytic infiltration in the connective tissue. Haematoxylin-eosin (H.-E.,) $\times 40$ (Fig. 1), × 200 (Fig. 2)
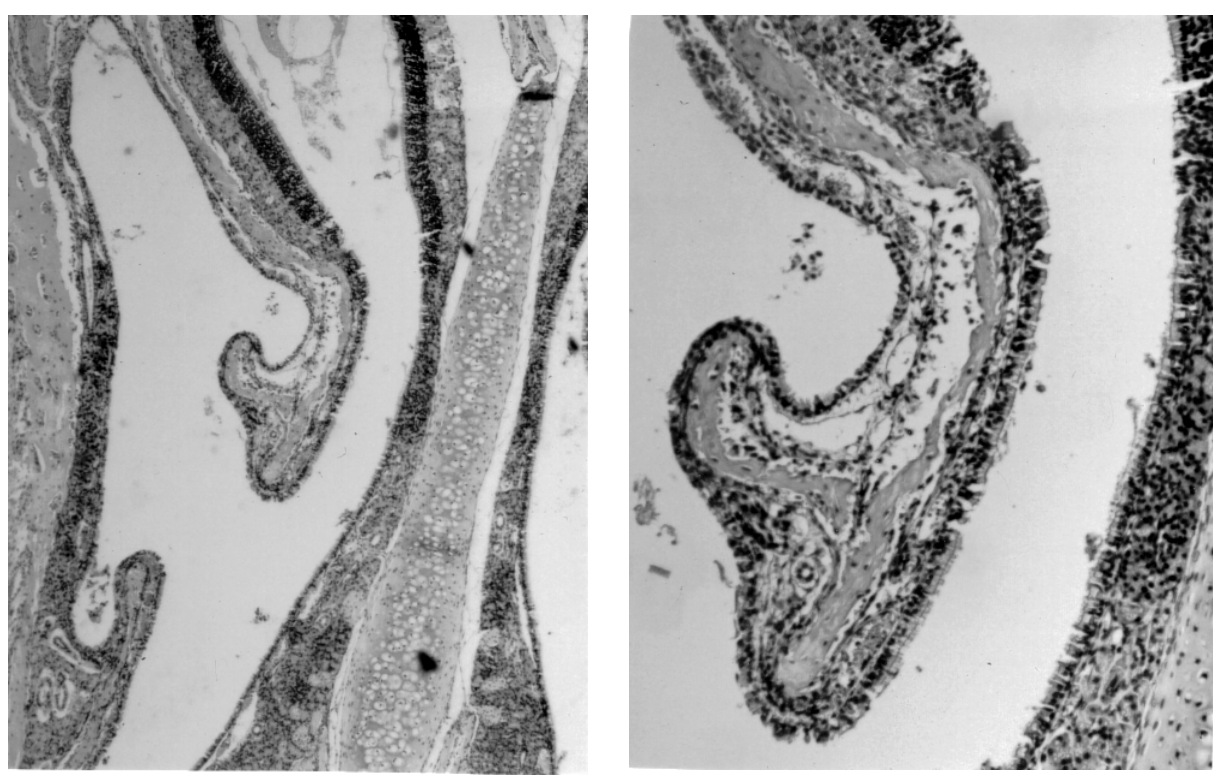

Figs 3 and 4. The nasal turbinates of a mouse infected with DNT-B58GP Bordetella bronchiseptica strain. Slight lympho-histiocytic infiltration (without other lesions). H.-E., $\times 40$ (Fig. 3), × 200 (Fig. 4) 

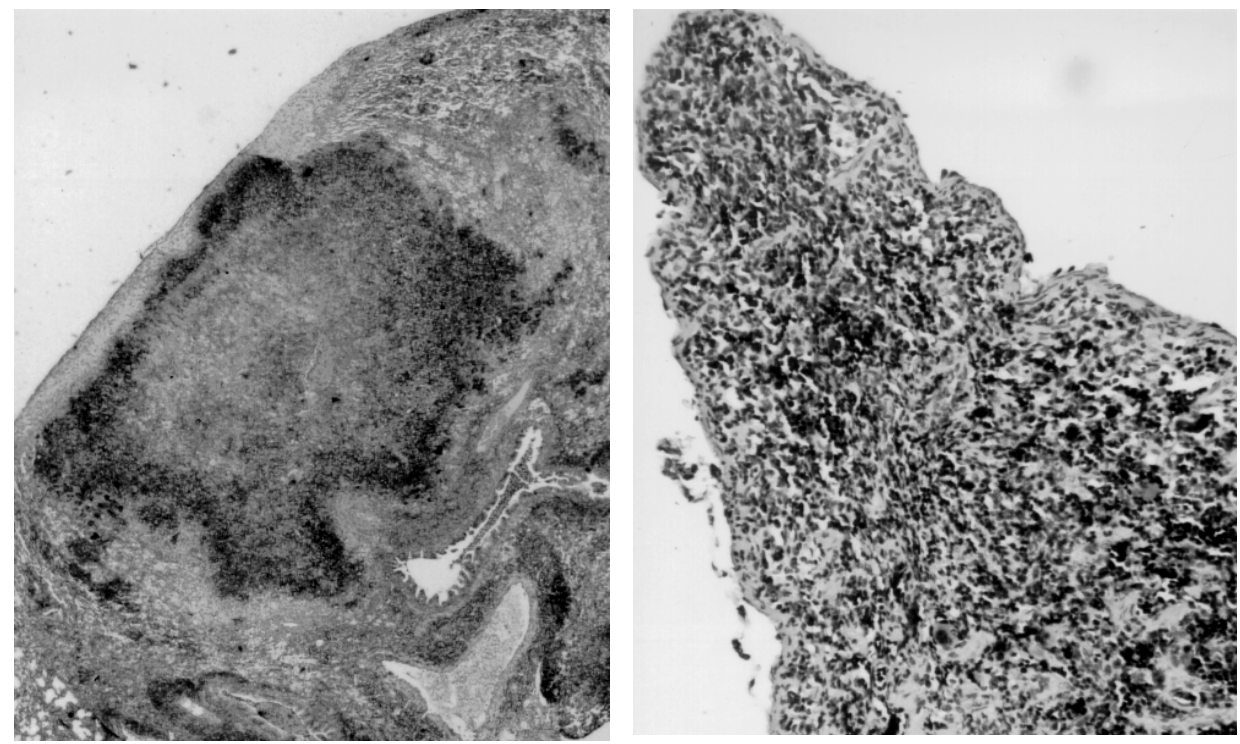

Fig. 5. Necrotic area surrounded with inflammatory reaction in the lungs of a mouse infected with DNT+ B58 Bordetella bronchiseptica strain. H.-E., $\times 40$

Fig. 6. Severe lymphocyte depletion (atrophy of Malpighian bodies) in the spleen of a mouse infected with DNT + B58 Bordetella bronchiseptica strain. H.-E., $\times 200$
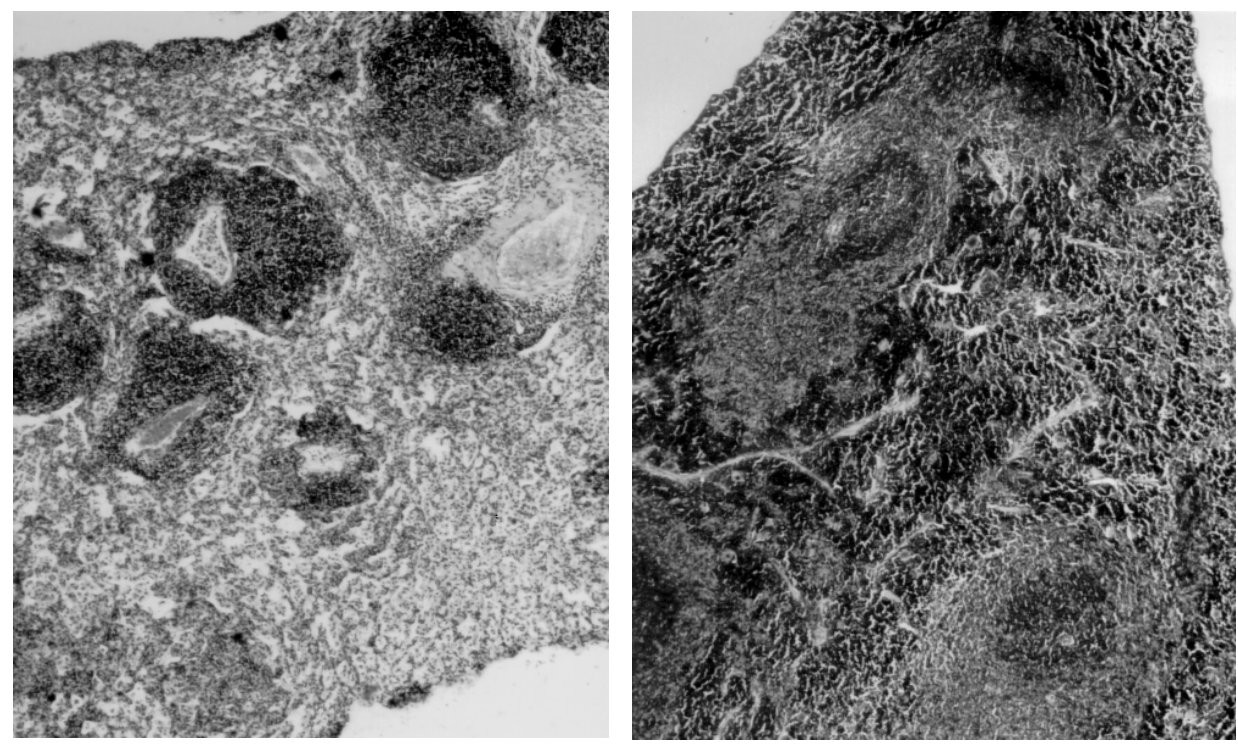

Fig. 7. Catarrhal pneumonia with characteristic lympho-histiocytic peribronchial and perivascular infiltration in the lung of a mouse infected with DNT- B58GP Bordetella bronchiseptica strain. H.-E., $\times 40$

Fig. 8. Lymphoid hyperplasia in the Malpighian bodies and cell-rich red pulp in the spleen of a mouse infected with DNT- B58GP Bordetella bronchiseptica strain. H.-E., $\times 40$ 
Turbinate atrophy was observed in mice infected with the DNT + strain, but not in those infected with the DNT- strain. Because of the small size of the nasal cavity, it was not possible to measure colonisation by the two B. bronchiseptica strains. The comparable lethality of the two B. bronchiseptica inoculated groups and the observation of signs of inflammation in the nasal mucosa of mice infected with strain B58GP make it likely that strain B58GP was present in the nasal cavity in sufficient numbers to produce comparable amounts of its other virulence factors. This indicates that DNT is the cause of turbinate atrophy in mice and not other factors produced by phase I strains of $B$. bronchiseptica.

Examination of the spleen confirmed that the B. bronchiseptica DNT has a lienotoxic effect while a stimulation of the lymphatic organs is seen in its absence as it was previously suggested (Magyar, 1990). It is of interest that DNT has been reported to suppress antibody responses in mice (Horiguchi et al., 1992). The immunosuppression was considered to be attributable to a dysfunction of the spleen that had been atrophied by DNT. Magyar and Glávits (1990) suggested that the so-called lienotoxic effect of B. bronchiseptica DNT is essentially a selective inhibition of the multiplication of cells belonging to the lymphoid, myeloid and platelet system. These findings underline the importance of B. bronchiseptica DNT in adversely altering the immune function of the host.

Both strains of $B$. bronchiseptica induced pneumonia in intranasally inoculated mice, though the pathology induced was fundamentally different. In mice infected with strain B58GP, catarrhal pneumonia with characteristic lympho-histiocytic peribronchial and perivascular infiltration was noticed. In mice infected with strain B58, large necrotic areas were seen surrounded by an inflammatory reaction. Thus DNT appears to be able to directly damage lung tissues, at least in mice. It seems that DNT production enhances the establishment of $B$. bronchiseptica in the lungs, presumably by reducing the local resistance and causing severe local damage to the lung tissues. Its importance to disease needs further elucidation although it seems probable that the production of DNT resulted in more pronounced systematic toxic effects. The role of DNT in swine also needs to be investigated.

\section{Acknowledgements}

The authors thank É. Hegedüs and Sz. Lakosi for their skilled technical assistance. This work was supported by grant OTKA T025536, and the Biotechnology and Biological Sciences Research Council grant (18/ICR07622) to AJL. 


\section{References}

Goodnow, R. A. (1980): Biology of Bordetella bronchiseptica. Microbiol. Rev. 44, 722-738.

Horiguchi, Y., Matsuda, H., Koyama, H., Nakai, T. and Kume, K. (1992): Bordetella bronchiseptica dermonecrotizing toxin suppresses in vivo antibody responses in mice. FEMS Microbiol. Lett. 69, 229-234.

Kielstein, P., Heilmann, P. and Magyar, T. (1987): Pasteurella multocida als Erreger der Rhinitis atrophicans: Bedeutung des Dermonecrotoxins im Babymaustest sowie In-vitro-Nachweis von Adhärenzeigenschaften. J. Vet. Med. B 34, 216-226.

Krüger, M. and Horsch, F. (1982): Untersuchungen zur Differenzierung von Bordetella bronchiseptica Stämmen. 1. Mitteilung: Nachweis des hitzenlabilen Exotoxins und des Haemagglutinins. Arch. Exp. Vet. Med. Leipzig 36, 691-698.

Magyar, T. (1990): Virulence and lienotoxicity of Bordetella bronchiseptica in mice. Vet. Microbiol. 25, 199-207.

Magyar, T. and Glávits, R. (1990): Immunopathological changes in mice caused by Bordetella bronchiseptica and Pasteurella multocida. Acta Vet. Hung. 38, 203-210.

Magyar, T., Chanter, N., Lax, A. J., Rutter, J. M. and Hall, G. A. (1988): The pathogenesis of turbinate atrophy in pigs caused by Bordetella bronchiseptica. Vet. Microbiol. 18, 135-146.

Magyar, T., Semjén, G. and Osváth, Zs. (1985): Investigation of adhesive and non-adhesive Bordetella bronchiseptica strains in a suckling-mouse model. Acta Vet. Hung. 33, 137-141.

Masuda, M., Betancourt, L., Matsuzawa, T., Kashimoto, T., Takao, T., Shimonishi, Y. and Horiguchi, Y. (2000): Activation of Rho through a cross-link with polyamines catalyzed by Bordetella dermonecrotizing toxin. EMBO J. 19, 521-530.

Pullinger, G. D., Adams, T. E., Mullan, P. B., Garrod, T. I. and Lax, A. J. (1996): Cloning, expression and molecular characterization of the dermonecrotic toxin gene of Bordetella spp. Infect. Immun. 64, 4163-4171.

Ridley, A. J. (1996): Rho: theme and variations. Current Biology 6, 1256-1264.

Roop, R. M., Veit, H. P., Sinsky, R. J., Veit, S. P., Hewlett, E. L. and Kornegay, E. T. (1987): Virulence factors of Bordetella bronchiseptica associated with the production of infectious atrophic rhinitis and pneumonia in experimentally infected neonatal swine. Infect. Immun. 55, 217-222.

Semjén, G. and Magyar, T. (1985): A bovine haemagglutinin of Bordetella bronchiseptica responsible for adherence. Acta Vet. Hung. 33, 129-136.

Sawata, A. and Kume, K. (1982): Nasal turbinate atrophy in young mice inoculated with Bordetella bronchiseptica of pig origin. Am. J. Vet. Res. 43, 1845-1847.

Weiss, A. A. and Goodwin, M. S. (1989): Lethal infection by Bordetella pertussis mutants in the infant mouse model. Infect. Immun. 57, 3757-3764. 